\title{
Bioengineering System for Prediction and Early Prenosological Diagnostics of Stomach Diseases based on Energy Characteristics of Bioactive Points with Fuzzy Logic
}

Riad Taha Al-Kasasbeh ${ }^{1 *}$, Nikolay Korenevskiy², Mahdi Alshamasin ${ }^{1}$ and Dmitry Klionskiy

${ }^{1}$ Faculty of Engineering Technology, Al-Balqa Applied University, Jordan

${ }^{2}$ Faculty of Biomedical Engineering, South West State University, Russia

Saint Petersburg Electrotechnical University "LETI", Saint Petersburg, Russian Federation

\begin{abstract}
We apply mathematical models for the interaction of the internal and biologically active points of meridian structures. Amongst the diseases for which reflex diagnostics are effective are those of the stomach disease. It is shown that use of fuzzy logic decision-making yields good results for the prediction and early diagnosis of stomach diseases, depending on the reaction energy of biologically active points (acupuncture points). It is shown that good results for the prediction and early diagnosis of diseases from the reaction energy of biologically active points (acupuncture points) are obtained by using fuzzy logic decision-making. Analysis of resistances lower and higher than the nominal values allowed us to specify the prognostic and diagnostic models.
\end{abstract}

Keywords: Acupuncture points; Fuzzy logic; Reflex diagnostics; Diagnostically important points (DIP); Confidence factors; Membership functions; Stomach diseases

\section{Introduction}

Research conducted by scientists from several academic communities has shown that one possible approach to prediction and early detection of various diseases, including stomach diseases, is reflex diagnostics. This approach is based on measuring energetic characteristics of biologically active points (BAPs) [1-12].

One of the advantages of reflex diagnostic methods is the fact that the response of BAPs to the change in the internal structure of the human body occurs prior to the clinical presentation of a disease. This allows us to detect and treat diseases in their earliest stages and sometimes even avert diseases by taking certain preventive measures. At the same time medical, technical, and time-related costs for arranging research are significantly lower in comparison with traditional approaches $[5,9,11,13,4-16]$

\section{Materials and Methods}

One of the important tasks connected with generating decisionmaking rules is informative feature selection and, in particular, selecting informative BAPs.

Selecting informative BAPs will be more effective if we take into account the way information about the condition of internal structures on these BAPs is displayed. The aforementioned features of "displaying" information include the transmission of a large volume of information (multiple diagnoses, symptoms, and syndromes) to one BAP; cyclic changes of the energetic condition of BAPs during the day even for the normal meridian energy balance; a large amount of data, which has to be analyzed if a pathology is unknown in advance [1,2,6,17-20].

Such features of information transmitted to BAPs impede the selection of informative points in the case of applying traditional methods adopted in the information theory and in the theory of pattern recognition.

Due to the existing peculiarities of representing information about the condition of the human body on BAPs, various methods and algorithms have been suggested $[5,15,18]$.
These methods and algorithms are intended to search for special combinations of BAPs. The analysis of such combinations makes it possible to confirm the diagnosis in question or to refute the diagnosis reflected in BAPs according to reference data, when the disease has not affected a person. The combinations described above are called "diagnostically important points" (DIPs) $[5,6,18]$.

Special research on prediction, early and differential diagnostics of cardiovascular system diseases, diseases of the digestive tract, nervous system diseases, musculoskeletal system diseases, diseases of the respiratory system, etc. has shown that the use of DIPs, in combination with other informative features, enables us to obtain decision rules providing high-quality classification [5,17,21-23].

Multiple investigations by scientists have demonstrated that projective zones and, in particular, BAPs are capable of accumulating and radiating different types of energy: thermal energy, electric energy, electromagnetic energy, etc. The amount of this energy can be determined using both electric and non-electric parameters [2$6,8,9,11-13,23,24]$.

When selecting parameters that characterize the energetic condition of BAPs we relied upon the accumulated experience and results of our own research. These results made it clear that from the point of view of instrumental resources, manufacturability, and reliability of what we have found, the most suitable parameters are the magnitudes of BAP electric resistance, measured in alternating current with a frequency

*Corresponding author: Riad TahaAl-Kasasbeh, Faculty of Engineering Technology, Al-Balqa Applied University, Jordan, Tel: +962777787972; E-mail: rjordanjo@mail.ru

Received September 09, 2015; Accepted October 02, 2015; Published December 12, 2015

Citation: Al-Kasasbeh RT, Korenevskiy N, Alshamasin M, Klionskiy D (2015) Bioengineering System for Prediction and Early Prenosological Diagnostics of Stomach Diseases based on Energy Characteristics of Bioactive Points with Fuzzy Logic. J Biosens Bioelectron 6: 182. doi:10.4172/2155-6210.1000182

Copyright: (c) 2015 Al-Kasasbeh RT, et al. This is an open-access article distributed under the terms of the Creative Commons Attribution License, which permits unrestricted use, distribution, and reproduction in any medium, provided the original author and source are credited.

Paper Presented at the 2nd Biomedical Engineering Conference and Expo November 30-December 01, 2015 San Antonio, USA 
of $1 \mathrm{kHz}$ and a current strength not exceeding $10 \mathrm{~mA} .[5,9,14-16,18]$.

We can find highly informative interval estimates of electric resistances $\Delta \mathrm{R}_{\mathrm{j}, \mathrm{k}}$ with the indicated upper and lower bounds of resistances for interval $k$ and point $j[5,9,14-16,18]$. The works mentioned above also say that relative deviations of the current values of resistances $\mathrm{R}_{\mathrm{j}}$ from their nominal values $\mathrm{R}_{\mathrm{inom}}$ are very informative from a medical point of view. These relative deviations are calculated by the following formula:

$$
\delta R j=\frac{|R j n o m-R j|}{R j n o m} \cdot 100 \% \text {. }
$$

When solving particular tasks with representative samples, it was established that if we fix measurement modes, sizes, and locations of electrodes and provide identical conditions for measuring both nominal and diagnostic values of electric resistances of BAPs, we will see that when changing the functional or health condition of an examined patient for the set of DIPs, it is possible to observe significant $\mathrm{p}<0.1$ deviations of the measured characteristics from their nominal values $[4,5,12-16,17-21,23]$.

The early (prenosological) stage of diseases is characterized by a decrease in DIP resistances of $20-30 \%$. Mild and moderate degrees of severity lead to a decrease in resistance of $30-60 \%$. The acute phase is distinguished by a decrease in resistance of more than $60 \%$ from the initial values. Severe forms of diseases connected with protracted processes are known to have more than $50 \%$ increases in the resistance of BAPs in comparison with their initial values. This, both increases and decreases have diagnostic and predictive significance.

Furthermore, it was established that in most cases the quality of diagnosis improves if, in addition to DIPs, other highly informative points connected with the diseases in question are used. As a rule these additional points are indicated by experienced experts, but they can also be found during statistical research, for example, using the Kullback technique [25].

According to the technique adopted in the decision-making theory, after selecting informative features (lists of informative BAPs including DIPs) we need to deal with the problem of choosing adequate mathematical techniques and generating the corresponding decision rules.

The experience of solving various prediction and diagnostic problems with the use of information about the energetic condition of projective zones and, in particular, BAPs has shown that the measurable indices are fuzzy and incomplete for the problem being discussed. Classes employed for decision-making (especially for prediction and early diagnostics) have fuzzy boundaries with overlapping areas, which migrate from one class to another $[6,19,23,26,27]$.

According to the recommendations [5,22,25-33] for generating decision rules it is reasonable to apply decision-making based on fuzzy logic.

The experience of using fuzzy logic for decision-making in medicine, psychology, ecology, and a number of other knowledge domains has confirmed that two areas of fuzzy logic have now become the most popular. These areas are briefly described below.

The first area is based on the fuzzy set theory, when sets are represented by certain membership functions $\mu_{A}(x)$ for fuzzy set $A$. The idea behind the construction of fuzzy sets is expounded in the works by Zade $[28,30-33,34]$.
The second area uses special confidence coefficients $\omega_{\ell}$ for hypotheses (predictions, diagnoses, etc.). These coefficients are obtained and calculated with the help of the algorithm suggested by Shortlif $[26,30]$.

In this paper both approaches are employed, but unlike the traditional fuzzy logic of decision-making developed by Zade and relying upon concepts and laws of fuzzy sets, in the present paper we use the concept "membership function" $\mu_{\omega_{\ell}}$ for the investigated classes of conditions $\omega_{\ell}$. These classes are continuous areas of a multi-dimensional feature space. The construction of fuzzy decision rules for the multidimensional feature space $\mathrm{X}=\left(\mathrm{x}_{1}, \ldots, \mathrm{X}_{\mathrm{i}}, \ldots \mathrm{x}_{\mathrm{n}}\right)$ is performed on the basis of aggregate operations. These operations are chosen depending on the structure of data used in an experiment. For the purpose of exploring the structure of multidimensional data and forming recommendations for choosing the type and parameters of membership functions, exploratory analysis is needed [5,18,19,24,28]. This analysis is also indispensable for finding ways of unifying these recommendations.

Several items can be clarified and discovered during the exploratory analysis. Among them are the possibility and reasonability of solving the recognition problem on the basis of its geometrical interpretation; the possibility of linear or piecewise-linear division of classes, existence of nested class structures of the type "ball in a ball", "ball in a cup", "ellipsoid in a ball"; existence of class overlapping areas, different types of areas and their structure, etc. [5,20,24,28].

Knowing various characteristics of class structure allows us to select the type of basic variables and characteristics of membership functions in a reasonable way; to obtain partial decision rules for subspaces belonging to the information feature space; to construct the final decision rule from partial decision rules, thus providing the required classification quality. It is important to emphasize that all the operations mentioned above are performed in a scientifically substantiated way due to the availability of class characteristics. The description of the general method needed for generating heterogeneous fuzzy decision rules on the basis of the exploratory analysis is provided in $[5,20,24,28]$. This method was developed for dealing with the problems of prediction and medical diagnostics.

In order to unify the synthesis of decision rules for diagnostic and prediction problems, the diagnostic problem is considered in this paper as a classification problem with two classes: the examined patient will not become ill in the next T years (class $\omega_{0}$ ); the examined patient will become ill in the next $\mathrm{T}$ years (class ).

When choosing informative BAPs and other indices, it is recommended to pay attention to those whose registration increases the confidence in hypothesis $\omega_{\ell}$ (illness) being investigated. According to the recommendations given in $[5,26,28]$ it is reasonable to select the storage iterative formula of E. Shortlif in its two modifications. This formula will be used as a basic one for calculating decision confidence on the basis of hypothesis $\omega_{\ell}$ :

$$
\begin{aligned}
& \mathrm{CC}_{\omega_{i}}(i+1)=\mathrm{CC}_{\omega_{i}}(i)+\mathrm{CC}_{\omega_{i}}^{*}\left(x_{i+1}\right)\left[1-\mathrm{CC}_{\omega_{i}}(i)\right] \\
& \mathrm{CC}_{\omega_{i}}(i+1)=\mathrm{CC}_{\omega_{i}}(i)+\mu_{\omega_{\ell}}\left(x_{i+1}\right)\left[1-\mathrm{CC}_{\omega_{i}}(i)\right]
\end{aligned}
$$

Where $\mathrm{CC}_{\omega_{\ell}}(i)$ is the confidence coefficient for hypothesis $\omega_{\ell}$ on condition that $i$ informative features have already been involved in hypothesis testing; $\mathrm{CC}_{\omega_{\ell}}^{*}\left(x_{i+1}\right)$ is the confidence coefficient in 
diagnosis (prediction) $\omega_{\ell} \omega_{1}$ when only one feature $x_{i+1}$ is used; is the membership function for class $\omega_{\ell}$ with a basic variable with number $i+1 \quad \mathrm{CC}_{\omega_{\ell}}(1)=\mu_{\omega_{\ell}}\left(x_{1}\right)$.

If experts have chosen interval estimates (resistance ranges) $\Delta R_{j, k}$ ( $j$ is the number of BAP, $k$ is the number of the range $\Delta R$ ) as the original information, formula (1) will be converted into the expression:

$$
\mathrm{CC}_{\omega_{\ell}}(j+1)=\mathrm{CC}_{\omega_{\ell}}(j)+\mathrm{CC}_{\omega_{\ell}}^{*}\left(\Delta R_{j+1, k}\right)\left[1-\mathrm{CC}_{\omega_{\ell}}(j)\right] \text {. }
$$

If preference is given to membership functions for hypotheses $\omega_{\ell}$ $\omega_{1}$, formula (2) will be modified:

$$
\mathrm{CC}_{\omega_{\ell}}(j+1)=\mathrm{CC}_{\omega_{\ell}}(j)+\mu_{\omega_{\ell}}\left(\delta R_{j+1}\right)\left[1-\mathrm{CC}_{\omega_{\ell}}(j)\right] .
$$

It was shown that due to the specific information represented by each BAP $[15,16,31]$, hypothesis $\omega_{\ell}$ can be confirmed with a large extent of confidence if and only if for all the points from the DIP list, their energetic characteristics (e.g., resistances) exceed the limits established. In other words, $\delta R_{j}$ exceeds the limit $\delta R_{j}^{t h r}$. This means that the onset of diagnostics using rule (2) must be carried out only under the following condition:

If [for all points Ylonging to DIPs for class $\omega_{\ell}$, resistance $\delta R_{j}$ exceeds the selected threshold $\left.\delta R_{j}^{t h r}\right]$ Then [it is necessary to determine the confidence in the diagnosis (prediction) $\omega_{\ell}$ ] Otherwise [confidence in diagnosis (hypothesis) $\omega_{\ell}$ is equal to zero].

Joining expression (4) with condition (5) we obtain the basic formula for determining confidence coefficients in the investigated diseases $\omega_{\ell}$ according to the response of BAPs:

$$
\begin{aligned}
& \text { If } \forall R_{j} \in[\mathrm{DIP}]_{\ell}: \delta R_{j} \geq \delta R_{j}^{\text {thr }} \text { Then } \\
& \mathrm{CC}_{\omega_{\ell}}(j+1)=\mathrm{CC}_{\omega_{\ell}}(j)+\mu_{\omega_{\ell}}\left(\delta R_{j+1}\right)\left[1-\mathrm{CC}_{\omega_{\ell}}(j)\right]
\end{aligned}
$$

Otherwise $\mathrm{CC} \quad 0$

Where $\forall$ is the universal quantifier, Expression (3) can be modified in a similar way.

The technical means used for registering BAP resistance was a special computer device, which exchanges data with a personal computer using the Bluetooth channel. The device operates in two modes: search mode for a point with acoustic, light, and graphical registration of BAPs and measurement mode on a frequency of $1 \mathrm{kHz}$ with a current strength of $5 \mathrm{uA}$.

In order to estimate the influence of physiological changes caused by stomach diseases upon the electrical resistance of BAPs connected with the investigated class of diseases, a special type of supervision was arranged. The supervision of patients, which lasted for three years, was organized in hospitals located in the town of Kursk (Russian Federation). During the supervision, the resistance of the selected BAPs was measured.

The patients participating in the study had to undergo an examination of stomach conditions. The patients' complaints were documented and when there were certain indications, fibrogastroduodenoscopy was performed. Those whose stomach pathology was not corroborated were assigned to class "healthy" $\left(\omega_{0}\right)$. Patients whose pathology was not confirmed during the initial visit, but who developed gastritis or ulcer in the next three years, were assigned to class $\omega_{R}$ - high risk of stomach diseases (prediction of stomach diseases). Patients who had early signs of gastritis or ulcer during the initial visit were assigned to class $\omega_{P}$ Using this technique training samples were formed containing 100 persons for each class. In This work it was established that decreasing and increasing the resistances of biologically active points relative to their nominal values has different influence on the decision-making process in stomach disease prediction problem. Analysis of resistances lower and higher than the nominal values allowed us to specify the prognostic and diagnostic models.

\section{Results}

The age of the examined patients varied from 18 to 70 years. The total number of men and women was the same (50 persons in each group). The analysis of the well-known meridians atlas showed that stomach diseases were connected with stomach meridian points, spleen-pancreas meridian points, bladder meridian points, etc. It is rather problematic to use all these points in practice and there is usually no medical need for doing this. For this reason after analyzing the whole list of points, it is reasonable to confine ourselves to the set of points applied in practice. In order to form this set we can use the opinion of experienced reflexologists and the availability of points selected for mining knowledge on the informativity of the explored BAPs. This informativity is calculated by techniques adopted in statistical analysis (e.g., Kullback statistical method [25]. The choice of informative BAPs with the subsequent synthesis of decision rules is made on the basis of the following technique. For a training set for all the extracted classes $\left(\omega_{0}, \omega_{R}, \omega_{P}\right)$ the average values of resistances were determined with the estimation of reliable differences between classes $P_{0 P}, P_{0 R}$. The points for which the reliable difference did not exceed 0.05 remained as candidates for becoming informative. Table 1 illustrates the prognostic and diagnostic possibilities of points $E 19$, E21, E22, E25, E36 [2].

This result coincides with those obtained in works $[5,14,18,34]$ concerning interconnections between stomach diseases, meridian BAPs and auricular points. The only difference is that the extension of the research area in this paper helped us to reveal two trends in the resistance change of BAPs concerning the nominal value $R_{j \text { ном }}$ (persons from class $\omega_{0}$ ). Some patients from classes' $\omega_{R}$ and $\omega_{P}$ are distinguished by reliable $(\mathrm{P}<0.05)$ decrease in BAP resistance relative to $R_{\text {jnom }}$ (except for point E22), which is characteristic of acute inflammatory processes according to previous research. Another category of patients had a reliable $(\mathrm{P}>0.05)$ increase in $\mathrm{BAP}$ resistance, which implies chronic protracted processes.

The crossover studies which have been conducted have shown that stable deviations in the resistance of informative BAPs from nominal values in both directions mean high probability $(\mathrm{P}<0.05)$ of emergence and development of the investigated digestive system diseases. In order to gain this probability we must isolate the groups with decreasing (upper values of the corresponding lines of classes' $\omega \ell$ in Table 1 ) and increasing (lower values of the corresponding lines of classes' $\omega \ell$ in

\begin{tabular}{|l|c|c|c|c|c|}
\hline \multirow{2}{*}{ Class } & \multicolumn{5}{|c|}{ Point } \\
\cline { 2 - 6 } & E19 & E21 & E22 & E25 & E36 \\
\hline \multirow{2}{*}{$\omega_{0}$} & 100 & 100 & 90 & 100 & 120 \\
\hline \multirow{2}{*}{$\omega_{R}$} & 61 & 65 & 72 & 63 & 83 \\
\hline \multirow{2}{*}{$\omega_{P}$} & 205 & 189 & 131 & 223 & 284 \\
\hline \multirow{2}{*}{$P_{0 P}$} & 58 & 49 & 81 & 54 & 72 \\
\hline$P_{0 R}$ & 0.05 & 0.05 & 0.10 & 0.05 & 0.01 \\
\hline
\end{tabular}

Table 1: average values of informative point resistances. The points are connected with stomach diseases. 
Citation: Al-Kasasbeh RT, Korenevskiy N, Alshamasin M, Klionskiy D (2015) Bioengineering System for Prediction and Early Prenosological Diagnostics of Stomach Diseases based on Energy Characteristics of Bioactive Points with Fuzzy Logic. J Biosens Bioelectron 6: 182. doi:10.4172/21556210.1000182

Page 4 of 9

Table 1) resistance values in a training set relative to $R_{\text {jnom }}$.

In view of the recommendations given, $[9,18,21]$ at the second stage of the research the resistance scales of BAPs were divided into 12 intervals with numbers $k$, for which diagnostic coefficients were calculated $[10,25]$ :

$$
\mathrm{DC}(\Delta R j, k)=10 \lg \frac{\left.P\left(\Delta R j, k^{/ \omega}\right)\right)}{P\left(\Delta R j, k^{/ \omega R}\right)},
$$

Where $P\left(\Delta R j, k^{/ \omega 0}\right)$ is the frequency of the $k$-th gradation of the $j$-th point in class $\omega_{0}$ (healthy); $P(\Delta R j, k / \omega R)$ is the frequency of the $k$-th gradation of the $j$-th point in class $\omega_{\mathrm{R}}$ (prediction of stomach disease).

Informativity of the $k$-th gradation of the $j$-th point $(I(\Delta R j, k))$ was determined with the use of the Kullback criterion [25]:

$$
I(\Delta R j, k)=\mathrm{DC}(\Delta R j, k) \cdot \frac{1}{2}\left[P\left(\Delta R j, k^{/ \omega} 0\right)-P\left(\Delta R j, k^{/ \omega} R\right)\right] .
$$

Informativity of the whole scale can be found by the expression

$$
I j=\sum_{k=1}^{n} I(\Delta R j, k) \text {. }
$$

In Table 2 there are results of diagnostic coefficient calculation, informativity for gradations and points for classes $\omega_{0}$ and $\omega_{R}$ in general.

In view of low informativity, point E22 was excluded from the list of informative BAPs.

For the rest of the points, experts had to transform the values of diagnostic coefficients into the values of confidence coefficients $\mathrm{CC}_{\omega_{R}}^{*}\left(\Delta R_{j, k}\right)$. This transformation should occur under the following conditions:

1) Class $\omega_{R}$ has negative values of $\operatorname{DC}(\Delta R j, k)$;

2) Maximal values of confidence coefficients do not exceed the confidence level (which is less than 1) for the points being used;

3) Zero value of a diagnostic coefficient corresponds to same confidence levels for classes' $\omega_{0}$ and $\omega_{\mathrm{R}}$;

4) Maximal positive values of $\operatorname{DC}(\Delta R j, k)$ that exceed the threshold values of these coefficients conform to zero values of the corresponding confidence coefficients.

For the conditions listed above, the table of confidence levels was formed at the expert level. The table is intended for predicting the investigated stomach diseases (Table 3 ). The elements of this table are partial confidence coefficients $\mathrm{CC}_{\omega_{R}}^{*}\left(\Delta R_{j, k}\right)$ for a prognostic formula like, for example, formula (3).

When forming this table, experts took into consideration the values of diagnostic thresholds for classes' $\omega_{0}$ and $\omega_{\mathrm{R}}$. The thresholds were calculated relative to $\mathrm{DC}(\Delta R j, k)$ according to the following formulas [17]:

$$
\text { Threshold } \omega 0=10 \lg \frac{1-\alpha}{\beta} \text {; Threshold } \omega 0=10 \lg \frac{1-\alpha}{\beta} \text {, }
$$

Where $\alpha$ is the allowable percentage of errors of the first kind

\begin{tabular}{|c|c|c|c|c|}
\hline Point & Range (kOhm) & $\ddot{A} \hat{E}(\Delta R j, k)$ & $I(\Delta R j, k)$ & ${ }^{I j} j$ \\
\hline 1 & 2 & 3 & 4 & 5 \\
\hline \multirow[t]{11}{*}{$E 19$} & $>500$ & -4 & 0,8 & \multirow[t]{11}{*}{25} \\
\hline & $400 \ldots 500$ & 0,9 & 0,05 & \\
\hline & $300 \ldots 399$ & 4 & 0,8 & \\
\hline & $200 \ldots 299$ & 6 & 1,8 & \\
\hline & 100...199 & 16 & 7,5 & \\
\hline & $90 \ldots 99$ & 16 & 7,5 & \\
\hline & $80 \ldots 89$ & 10 & 4 & \\
\hline & $70 \ldots . .79$ & 5 & 1,25 & \\
\hline & $60 \ldots 69$ & 2 & 0,2 & \\
\hline & $50 \ldots 59$ & -1 & 0,05 & \\
\hline & $<50$ & -4 & 0,8 & \\
\hline \multirow[t]{11}{*}{ E21 } & $>500$ & -6 & 1,2 & \multirow[t]{11}{*}{18} \\
\hline & $400 \ldots 500$ & -1 & 0,05 & \\
\hline & $300 \ldots 399$ & 0,9 & 0,05 & \\
\hline & $200 \ldots 299$ & 4 & 0,8 & \\
\hline & $100 \ldots 199$ & 13 & 5,8 & \\
\hline & $90 \ldots 99$ & 13 & 5,8 & \\
\hline & $80 \ldots 89$ & 6 & 1,8 & \\
\hline & $70 \ldots .79$ & 3 & 0,45 & \\
\hline & $60 \ldots 69$ & 0 & 0 & \\
\hline & $50 \ldots 59$ & -4 & 0,8 & \\
\hline & $<50$ & -6 & 1,2 & \\
\hline \multirow[t]{15}{*}{$E 22$} & $>500$ & -4 & 0,8 & \multirow[t]{11}{*}{4,85} \\
\hline & $400 \ldots 500$ & -2 & 0,2 & \\
\hline & $300 \ldots 399$ & 0 & 0 & \\
\hline & $200 \ldots 299$ & 2 & 0,2 & \\
\hline & 100...199 & 6 & 1,2 & \\
\hline & $90 \ldots 99$ & 6 & 1,2 & \\
\hline & $80 \ldots 89$ & 2 & 0,2 & \\
\hline & $70 \ldots .79$ & 0,9 & 0,05 & \\
\hline & $60 \ldots 69$ & 0 & 0 & \\
\hline & $50 \ldots 59$ & -2 & 0,2 & \\
\hline & $<50$ & -4 & 0,8 & \\
\hline & $>500$ & -6 & 1,2 & \\
\hline & $400 \ldots 500$ & -2 & 0,2 & \\
\hline & $300 \ldots 399$ & 0 & 0 & \\
\hline & $200 \ldots 299$ & 2 & 0,2 & \\
\hline \multirow[t]{7}{*}{$E 25$} & $100 \ldots 199$ & 16 & 7,5 & \multirow[t]{7}{*}{21} \\
\hline & $90 \ldots 99$ & 16 & 7,5 & \\
\hline & $80 \ldots 89$ & 4 & 0,8 & \\
\hline & $70 \ldots 79$ & 0,9 & 0,05 & \\
\hline & $60 \ldots 69$ & -2 & 0,2 & \\
\hline & $50 \ldots 59$ & -5 & 1,25 & \\
\hline & $<50$ & -7 & 2,45 & \\
\hline \multirow[t]{11}{*}{$E 36$} & $>500$ & -7 & 2,45 & \multirow[t]{11}{*}{25} \\
\hline & $400 \ldots 500$ & -4 & 0,8 & \\
\hline & $300 \ldots 399$ & 0,8 & 0,05 & \\
\hline & $200 \ldots 299$ & 2 & 0,2 & \\
\hline & $100 \ldots 199$ & 17 & 8 & \\
\hline & $90 \ldots 99$ & 16 & 7,5 & \\
\hline & $80 \ldots 89$ & 3 & 0,45 & \\
\hline & $70 \ldots . .79$ & 0 & 0 & \\
\hline & $60 \ldots 69$ & -3 & 0,45 & \\
\hline & $50 \ldots 59$ & -6 & 1,2 & \\
\hline & $<50$ & -9 & 3,6 & \\
\hline
\end{tabular}
when hypothesis $\omega_{0}$ is taken for hypothesis $\omega_{R} ; \beta$ is the allowable percentage of errors of the second kind when hypothesis $\omega_{R}$ is taken for hypothesis $\omega_{0}$.
Table 2: Informative BAPs used for predicting the investigated classes of stomach diseases. 
Citation: Al-Kasasbeh RT, Korenevskiy N, Alshamasin M, Klionskiy D (2015) Bioengineering System for Prediction and Early Prenosological Diagnostics of Stomach Diseases based on Energy Characteristics of Bioactive Points with Fuzzy Logic. J Biosens Bioelectron 6: 182. doi:10.4172/21556210.1000182

Page 5 of 9

\begin{tabular}{|c|c|c|c|c|c|c|c|c|c|c|c|c|}
\hline \multirow{2}{*}{$\begin{array}{l}\frac{8}{0} \\
\frac{\pi}{U}\end{array}$} & \multirow[t]{2}{*}{ BAP } & \multicolumn{11}{|c|}{ Resistance ranges, kOhm } \\
\hline & & $>500$ & $\begin{array}{c}400 \ldots \\
500\end{array}$ & $\begin{array}{c}300 \ldots \\
399\end{array}$ & $\begin{array}{c}200 \ldots \\
299\end{array}$ & $\begin{array}{c}100 \ldots \\
199\end{array}$ & $\begin{array}{c}90 \ldots \\
99\end{array}$ & $\begin{array}{c}80 \ldots \\
89\end{array}$ & $\begin{array}{c}70 \ldots \\
79\end{array}$ & $\begin{array}{c}60 \ldots \\
69\end{array}$ & $\begin{array}{c}50 \ldots \\
59\end{array}$ & $<50$ \\
\hline \multirow[t]{4}{*}{$\boldsymbol{\omega}$} & $E^{\star} 19$ & 0.6 & 0.5 & 0.35 & 0.2 & 0 & 0 & 0.2 & 0.3 & 0.45 & 0.55 & 0.6 \\
\hline & $E^{\star} 21$ & 0.65 & 0.6 & 0.5 & 0.25 & 0 & 0 & 0.2 & 0.35 & 0.5 & 0.6 & 0.65 \\
\hline & $E^{\star} 25$ & 0.65 & 0.6 & 0.5 & 0.25 & 0 & 0 & 0.25 & 0.5 & 0.6 & 0.65 & 0.7 \\
\hline & E36 & 0.7 & 0.6 & 0.45 & 0.2 & 0 & 0 & 0.25 & 0.5 & 0.65 & 0.8 & 0.9 \\
\hline
\end{tabular}

* - points from the list of DIPS

Table 3: Partial confidence coefficients for the prediction of stomach diseases.

\begin{tabular}{|c|c|c|c|c|c|c|c|c|c|c|c|c|c|c|c|c|c|c|c|c|c|c|c|c|}
\hline $\begin{array}{ll} & X_{i} \\
Y_{j} & \end{array}$ & 1 & 2 & 3 & 4 & 5 & 6 & 7 & 8 & 9 & 10 & 12 & 13 & 14 & 15 & 16 & 17 & 18 & 19 & 20 & 21 & 22 & 23 & 24 & 25 \\
\hline E19 & 0 & 0 & 1 & 1 & 1 & 1 & 1 & 1 & 1 & 1 & 0 & 0 & 0 & 0 & 0 & 0 & 0 & 0 & 0 & 0 & 0 & 0 & 0 & 0 \\
\hline E21 & 1 & 1 & 1 & 0 & 0 & 0 & 0 & 0 & 0 & 0 & 0 & 0 & 0 & 0 & 0 & 0 & 0 & 0 & 0 & 0 & 0 & 0 & 0 & 0 \\
\hline E25 & 1 & 0 & 0 & 0 & 0 & 0 & 1 & 0 & 0 & 0 & 1 & 1 & 1 & 1 & 1 & 0 & 0 & 0 & 0 & 0 & 0 & 0 & 0 & 0 \\
\hline E36 & 1 & 0 & 0 & 0 & 0 & 0 & 1 & 0 & 0 & 0 & 0 & 1 & 0 & 0 & 0 & 1 & 1 & 1 & 1 & 1 & 1 & 1 & 1 & 1 \\
\hline
\end{tabular}

Table 4: Binary table of connections concerning stomach diseases.

Having taken $\alpha=\beta=0.95$ the threshold values \pm 13 were obtained.

The location of the points selected for the prediction of stomach diseases is shown in Figure 1.

The lists of DIPs for the "inclusion" of a fuzzy prognostic decision rule are formed according to the recommendations given in works $[5,18]$. Following these recommendations taken from the well-known meridian atlases, for example [35], all the cases connected with the selected points $\mathrm{X}_{0}$ are considered - stomach ulcer, acute and chronic gastritis.

E21- a typical point: X1- diarrhea; X2- spastic pain in the gut; X3loss of appetite.

E22- a typical point: $\mathrm{X} 1 ; \mathrm{X} 3$; - as above; $\mathrm{X} 4$ - pain and rumbling in the stomach; X5- pain around the navel; X6- violation of the peristaltic intestine; $\mathrm{X} 7$ - asthma.

E19- a typical point: X3; - as above; X8- liver disease; X9- lung disease; X10- pain in epigastric areas; X11- vomiting; X12- coughing, shortness of breath; X13- pain in the chest and back; X14- bronchial and cordial asthma.

E25- a signal point of the meridian of the colon: X1, X5, X7, X11; - as above; X15- dysentery; X16- constipation; X17- cholecystitis; X18conversion disorder; X19- endothermy.

E36- a point with a wide spectrum of action: X1,X11,X16; - as above; X20- pain in epigastric areas;X21- bloating; X22- enteritis; X23pain in the knee joint and shin; X24- eye diseases; X25- fever; X26enuresis; X27- arterial hypertensia; X28- asthenic condition [5] (Table 4).

The analysis of this table shows that three points E19, E21 and E25 provide the exclusion of all the interfering factors because the product of the columns for these points is equal to zero. These points are available for measuring and are recommended as DIPs. Point E21 has been excluded by experts as uninformative. It has been suggested that point E36 be used as an additional feature because of its high informativity (Figure 1) [2].

Owing to the list of DIPs we have obtained and the calculation results given in Tables 2 and 3, formula (6) can be modified the following way:
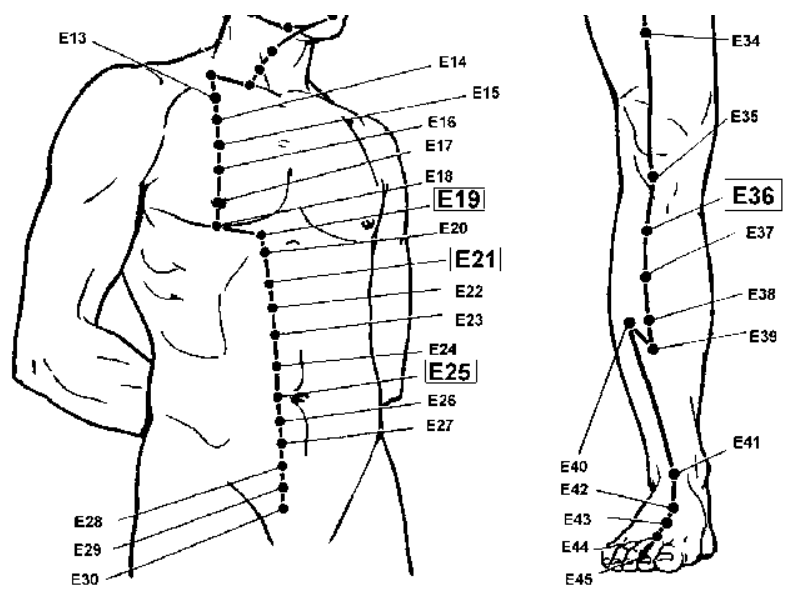

Figure 1: Location of the selected points on the stomach meridian.

If

$\{[(R E 19>200 \mathrm{kOm})$ AND $(R E 21>200 \mathrm{kOm})$ AND $(R E 25>200 \mathrm{kOm})]$

OR $[(R E 19<80 \mathrm{kOm})$ и $(R E 21<80 \mathrm{kOm})$ и $(R E 25<80 \mathrm{kOm})]\}$ Then

$\left\{\mathrm{CC} \omega_{R}(j+1)=\mathrm{CC} \omega_{R}(j)+\mathrm{CC}^{*} \omega_{R}(\Delta R j+1, k)\left[1-\mathrm{CC} \omega_{R}(j)\right]\right\}$

Otherwise $\mathrm{CC} \omega_{R}=0$

Where, $\mathrm{CC} \omega_{R}{ }^{(1)=\mathrm{CC}^{*}}{ }^{*}(\Delta R E 19, k) R_{2, k}=R_{E 21, k} ; R_{3, k}=R_{E 25, k} ; R_{4, k}=R_{E 36, k}$

As a result of mathematical modeling it was established that for maximal values of partial confidence coefficients, the final confidence in the prediction of the onset of stomach diseases defined by formula (10) exceeds 0.95 .

For the most frequent values of resistance on condition of the stable deviation of BAPs resistance from nominal values ("range" 90 $\mathrm{kOhm}, 200 \mathrm{kOhm}$ ) the value $\mathrm{CC}_{\omega_{R}}$ exceeds 0.85 .

A peculiarity of the energetic characteristics of BAPs and a number of other informative features is the fact that they can change under 
the influence of one-time, relatively short, perturbing internal and external impacts. After that, if the organism has sufficient adaptation potential, all the features can return to the range of allowable values or stay beyond the scope of the values considered to be normal for a rather long period of time (week, month, year, etc.)

The first case more rarely indicates abnormal deviation of a human body condition, while the second case usually means a high risk of the emergence and development of new diseases, or the diseases that have already affected a person. The longer the time period, in which we can observe the deviation of energetic characteristics from their nominal values, the more confidently we can speak about hypothesis $\omega \ell$.

It is possible to take into account the duration of energetic characteristic deviation of BAPs from the corresponding nominal values. For this purpose we need formulas for calculating confidence coefficients in hypotheses $\omega \ell$. It is also necessary to introduce different confidence levels. For example, the first confidence level with its partial decision rules can be linked with conclusions drawn as a result of onetime measurements; the second confidence level can be linked with the results obtained in the presence of a stable trend in measurable parameters exceeding nominal values during the week; the third level exceeding nominal values during two weeks, the fourth one month, etc.

Multiple methods are suitable for estimating the time when electrical characteristics of BAPs are kept beyond the nominal values.

For example, the confidence coefficient for different confidence levels $r$ can be determined by multiplying the confidence coefficient for the first confidence level $\mathrm{CC} \omega_{\ell}^{1}$ by the weight coefficient $\gamma_{\omega_{\ell}}\left(D j, t_{j}^{*}\right)$, which depends on the outcome value of the measured resistance $R$ beyond the nominal values $D_{j}$ and the time $t_{j}^{*}$ during which $R_{j}$ is kept beyond the nominal value. The corrected confidence coefficient is determined by the expression:

$$
\mathrm{CC}^{r} \omega_{\ell}{ }^{\gamma} \omega_{\ell}{ }^{(D j, t j) \cdot \mathrm{CC}} \omega_{\ell}
$$

When choosing the values $\gamma \omega_{\ell}(D j, t j)$, it is necessary to remember that

$$
\mathrm{CC}^{r} \omega_{\ell}<1
$$

Another method is to introduce, in addition to $\mu_{\omega_{\epsilon}}\left(\delta R_{j}\right)$, a membership function $\mu_{\omega_{t}, R_{j}}(t)$ for clarifying the term "critical time of a feature location beyond nominal values". The current value of this function calculated for a particular time during which we can observe the deviation of the resistance $R_{j}$ from the nominal value can be used for correcting the corresponding confidence coefficient. The improved confidence coefficient can be calculated by the formula:

$$
\mathrm{CC}^{*} \omega_{\ell}, j=\left\{\begin{array}{l}
0, \text { if } \mu \omega_{\ell}(\delta R j) \cdot \mu \omega_{\ell}, R_{j}{ }^{(t)=0} \\
\mu \omega_{\ell}(\delta R j)+\mu \omega_{\ell}, R_{j}{ }^{(t)}\left[1-\mu \omega_{\ell}(\delta R j)\right], \text { if } \mu \omega_{\ell}(\delta R j) \cdot \mu \omega_{\ell}, R_{j}{ }^{(t)>0}
\end{array}\right.
$$

In this expression we can see the growth of confidence in $\omega \ell$ with the increasing duration of the BAP energetic characteristic deviation with number $j$ from its nominal value with ${ }^{\mathrm{CC}^{*}}{ }_{\omega_{\ell}, j}$ tending to some upper bound. This bound does not exceed 1 according to the law determined by the parameters of the corresponding membership functions.
The given values of the confidence coefficients were obtained after expert evaluation and mathematical modeling. For a more objective evaluation of a decision-making quality, testing sets were formed and several quality indices were calculated. These indices comprise diagnostic sensitivity, diagnostic specificity, prognostic significance of positive and negative results.

Concerning stomach disease prediction, patients from the emergency hospital in Kursk (Russian Federation) were supervised. At the beginning of the research all the examined patients had to have the resistance of points E19,E21,E25, and E36 measured. The testing sets were obtained the following way. Those patients who were not suffering from stomach diseases were assigned to class $\omega_{\mathrm{R}}$. For each class, 100 persons were selected. After one year of medical supervision the accuracy of rule (10) was tested for threshold $\mathrm{CC}_{\omega_{R}}^{\mathrm{Thr}}=0,5$. The prognostic quality indices were calculated. Finally, using information about classification errors it was established that

$$
\mathrm{PZ}+=\mathrm{n} 1 /(\mathrm{n} 1+\mathrm{n} 2)=0,86 ; \mathrm{PZ}-=\mathrm{n} 3 /(\mathrm{n} 4+\mathrm{n} 3)=0,82 \text {. }
$$

In the latter expressions $n_{1}$ is the number of sick persons who have been classified correctly by a decision rule; $n_{2}$ is the number of healthy persons who have been classified by a decision rule as sick; $n_{3}$ is the number of healthy persons who have been classified correctly by a decision rule; $n_{4}$ is the number of sick persons who have been classified by a decision rule as healthy persons.

When modifying expression (10) due to time amendments (expression 11) the value PZ+ is increased to 0.92 ; the value PZ- is increased to 0.87 .

Thus, it was concluded and statistically proven that BAPs E19, E21, $E 25$, and $E 36$ can be used in practical medicine for stomach disease prediction.

The points selected for prediction were investigated as informative features for diagnosing early stomach diseases (class $\omega_{p}$ ). As in prediction tasks, the points under concern were used for determining diagnostic coefficients $\mathrm{DC}(\Delta R j, k)$ and Kullback informativity. The analysis of the results made it clear that unlike the prediction problem, diagnostic coefficients almost linearly and uniformly increase with the deviation of the investigated resistances of BAS from the nominal values provided in Table 1. For this reason and also due to the results obtained during the creation of fuzzy diagnostic decision rules for other diseases (described in [5,14-16,18-21,23]), we decided to choose membership functions for classes $\omega_{\mathrm{R}}$ and $\omega_{P}$ (with basic variables $\delta R_{j}$ ) as the basic elements of decision rules.

To determine the type and parameters of membership functions

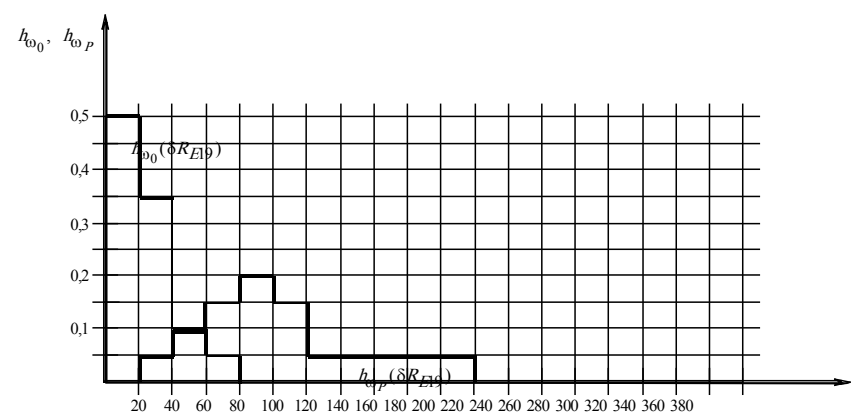

Figure 2: Histogram for classes $\omega_{0}$ and $\omega_{\mathrm{p}}$ using the scale $\delta R_{A 19}$ 
Citation: Al-Kasasbeh RT, Korenevskiy N, Alshamasin M, Klionskiy D (2015) Bioengineering System for Prediction and Early Prenosological Diagnostics of Stomach Diseases based on Energy Characteristics of Bioactive Points with Fuzzy Logic. J Biosens Bioelectron 6: 182. doi:10.4172/21556210.1000182

Page 7 of 9

${ }^{\mu} \omega_{R}(\delta R j)$ according to the recommendations, [6] histograms for classes $\omega_{0}$ and $\omega_{P}$ were constructed (using scale $\delta R_{j}$ ). The example of histograms for classes $\omega_{0}$ and $\omega_{P}$ is given in Figure 2 .

When proceeding from histogram images for classes to the corresponding membership functions, it was taken into account that the magnitude of a histogram reflects the relative frequency of different values $\delta R_{j}$ in class $\omega$. The value $\mu_{\omega_{P}}(\delta R j)$ reflects the confidence in hypothesis $\omega_{P}$ for each value of $j$.

Furthermore, it is necessary to remember that when including ${ }^{\mu} \omega_{P}(\delta R j)$ in a decision rule for checking hypothesis $\omega_{\mathrm{P}}$, the general confidence should not exceed a confidence value produced by experts for the investigated problem if only electrical characteristics of BAPs are used as informative features.

The algorithm operates according to the modified expression (6)

If $[(\delta R E 19>20 \%)$ AND $(\delta R E 21>20 \%) \quad$ AND $(\delta R E 25>20 \%)]$

Then $\left.\left\{\mathrm{CC} \omega_{P}(j+1)=\mathrm{CC} \omega_{P}(j)+\mu \quad \omega_{P}{ }^{(\delta R j+1)[1-\mathrm{CC}} \omega_{P}{ }^{(j)}\right]\right\}$

Otherwise $\mathrm{CC} \omega_{P}=0$

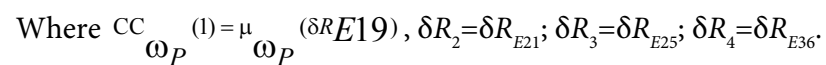

The plots of the corresponding membership functions found by experts are shown in Figure 3.

These plots correspond to the following analytical expressions:

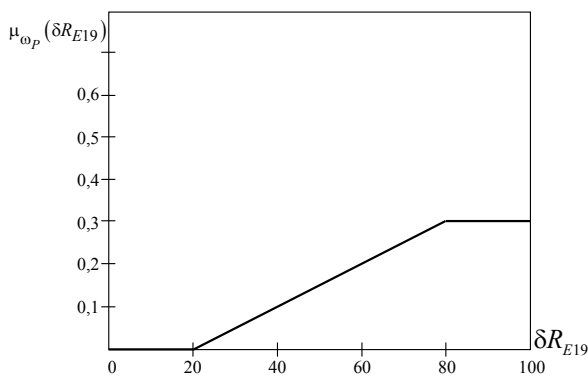

a)

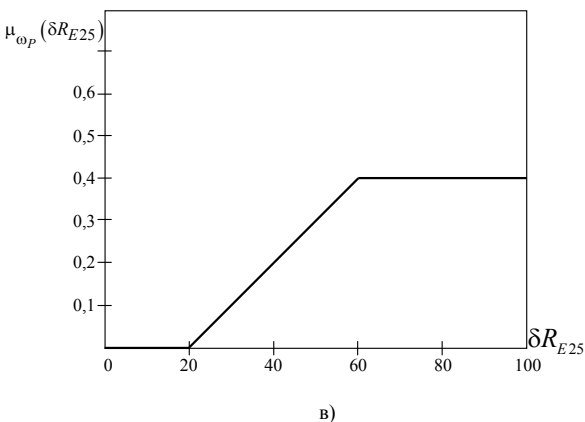

$\mu \omega_{P}(\delta R E 19)=\left\{\begin{array}{l}0, \text { if } \delta R E 19 \leq 20 \% ; \\ 0,005 \cdot \delta R E 19-0,1, \text { if } 20 \%<\delta R E 19 \leq 80 \% ; \\ 0,3, \text { if } \delta R E 19>80 \% .\end{array}\right.$

$\mu \quad(\delta R E 21)=\left\{\begin{array}{l}0, \text { if } \delta \quad 21 \leq 20 \% ; \\ 0,01 \cdot \delta R E 21-0,2, \text { if } 20 \%<\delta R E 21 \leq 60 \% ; \\ 0,4, \text { if } \delta \quad 21>60 \% .\end{array}\right.$

${ }^{\mu} \omega_{P}(\delta R E 25)=\left\{\begin{array}{l}0, \text { if } \delta R E 25 \leq 20 \% ; \\ 0,01 \cdot \delta R E 25-0,2, \text { if } 20 \%<\delta R E 25 \leq 60 \% ; \\ 0,4, \text { if } \delta R E 25>60 \% .\end{array}\right.$

$\mu \omega_{P}(\delta R E 36)=\left\{\begin{array}{l}0, \text { if } \delta R E 36 \leq 20 \% ; \\ 0,02 \cdot \delta R E 36-0,4, \text { if } 20 \%<\delta R E 36 \leq 50 \% ; \\ 0,6, \text { if } \delta R E 36>50 \% .\end{array}\right.$

By means of mathematical modeling it was found that for maximal values of all the membership functions the value of the confidence coefficient determined by expression (13) reaches 0.9 for the most frequent values of BAP resistances $\mathrm{CC}_{\omega_{P}}=0,87$.

For statistical evaluation of the decision rule quality two testing sets were formed: $\omega_{0}$ is the class of persons who do not have stomach diseases; $\omega_{P}$ is the class of persons who have an early stage of stomach diseases. Patients were diagnosed with the use of traditional methods of examination (fibrogastroduodenoscopy), without considering electrical characteristics of BAPs.

As a result of checking for the threshold $\mathrm{CC}_{\mathrm{Thr}}=0,5$ the following results were found: DSen $=n_{1} / n_{P}=0,91 ; \mathrm{DSpec}=n_{1} / n_{0}=0,89$, where $n_{P}$ $=n_{0}=100$ is the number of people in a testing set in classes $\omega_{0}$ and $\omega_{P}$ respectively $[2,5]$.

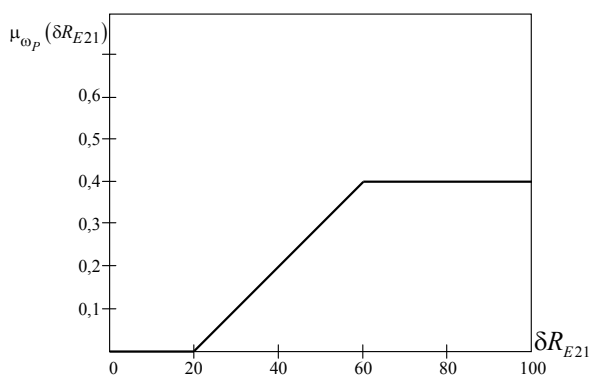

б)

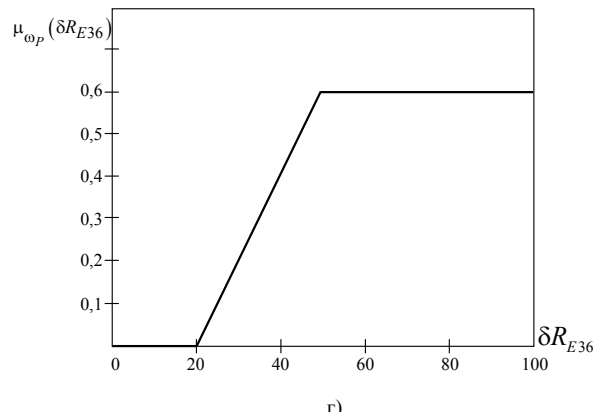

Figure 3: Plots of the membership functions: a) $\left.\mu \omega_{\mathrm{p}}{ }^{\mu} \omega_{P}{ }_{P}^{(\delta R E} E 19\right)$ with a basic variable $\delta R_{E 19} ;$ б) ${ }^{\mu} \omega_{P}{ }^{(\delta R} E 21$, with a basic variable $\delta R_{E 21}$; в) $\left.\mu{ }^{\mu} \omega_{P}{ }^{(\delta R E} E 25\right)$ with a basic variable $\left.\delta R_{E 25} ; \Gamma\right){ }^{\mu} \omega_{P}{ }^{(\delta R E 36)}$ with a basic variable $\delta R_{E 36}$ for the class «early stage of stomach diseases» (class $\left.\omega_{P}\right)$. 
When using correcting time amendments (rule (11)) the indices of diagnostic sensitivity and specificity exceed 0.95 .

The outcome of the research can be summarized as follows: first, a rather good coincidence between expert evaluation, mathematical modeling, and statistical tests was shown; second, when the corresponding quality indices are rather high, it is possible to recommend the results from the paper for further investigation and application [5].

\section{Discussion}

In contemporary medical practice there is a growing interest in traditional oriental methods of reflexodiagnostics and relexotherapy based upon the teaching about meridian energy balance of the body.

However, the fact that a large number of reflexotherapists use a traditional oriental conceptual apparatus, which does not coincide with contemporary medical terminology of anatomy, physiology, and biophysics diminishes medical confidence in this area of medical practice.

Another obstacle in the way of a wider and more effective use of reflexodiagnostics and reflexotherapy techniques is the lack of well-developed theoretical fundamentals that reveal the interaction mechanism of internal organs with meridian structures located on the surface of a human body. This problem dramatically decreases the potential capabilities of acupuncture. Furthermore, by using theoretical uncertainty, a great number of practicing reflexotherapists are known to exaggerate the potential of acupuncture, which leads to partial errors and discredits the corresponding approach to diagnostics and treatment.

The problems specified in the paper, as well as a number of other problems of acupuncture are eliminated to a large extent due to the research results obtained by the team of authors in Russia, Germany, and Jordan. All this prolonged and laborious work was made possible thanks to the financial support provided by the DFG fund. During the research several new mathematical models were offered. These models reflect the interaction between internal organs and BAPs of meridian structures: table models, models on the basis of the graph theory, models obtained by the automatic control theory methods, and multilevel models.

Analysis of the models mentioned above allowed us to specify the range of diseases for which reflexodiagnostics and reflexotherapy are the most effective methods. Computer implementation of such models provides precise solution of prediction tasks and helps experts achieve an early diagnosis of fundamental diseases, combined diseases, and coexistent diseases, when clinical manifestations are almost unobservable or undetectable by other techniques. When the diagnosis has already been established, the analysis of meridian models enables us to specify the degree of severity, prediction of the exacerbation and possible outcome of a disease. At the stage of reflexotherapy, the analysis of energy imbalance of meridian models makes it possible to construct rational schemes of impact on BAPs excluding harmful side effects on different organs and systems, which are in complex hierarchical interaction with an impact point.

A rather large number of prediction and diagnostic tasks, which are solved on the basis of information about the energetic condition of projective zones and, in particular, BAPs, showed that the measurable indices are fuzzy and incomplete for the problems under consideration. Classes used for decision-making (especially for prediction and early diagnostics) have fuzzy boundaries with overlapping areas, which migrate from one class to another. In these conditions for the purpose of creating the corresponding decision rules, it is reasonable to use the fuzzy logic theory of decision-making, when energetic characteristics of BAPs used for decision-making for class $\omega_{1}$ are represented by membership functions or partial confidence coefficients relative to the considered hypothesis $\omega_{1}$.

In this paper we have shown that the best quality of decisionmaking is provided if the synthesis of fuzzy decision rules is carried out according to exploratory analysis intended for studying various features of data. Then these features contribute to the choice of the form and parameters of partial decision rules (membership functions of Zade and confidence coefficients of E. Shortlif) and the methods of transformation in final decision-making rules.

By means of numerous investigations it was shown that projective zone and, in particular, BAPs are capable of accumulating and radiating different types of energy: thermal, electrical, electromagnetic, etc. The amount of this energy can be determined via electrical and non-electrical parameters.

By choosing parameters characterizing the energetic condition of BAPs we proceeded from the experience we have gained and our own findings. They indicated that from the point of view of instrumental errors, manufacturability, and reliability of the results, the most suitable parameters are electrical resistances of BAPs measured in alternating current of low frequency.

This paper contains information about the way the prediction problem can be solved and how it is possible to deal with the need for early diagnosis of stomach diseases using the suggested information processing techniques. Checking on testing sets with 100 persons for each class formed by highly-qualified experts showed that the diagnostic effectiveness of the solution exceeds 0.85 . Such a result permits us to recommend the decision rules we have developed for practical application. Furthermore, with the use of the suggested models and techniques of constructing fuzzy decision rules, the following problems have been solved: prediction, diagnosis, and prophylaxis of various diseases that affect the respiratory system, the digestive system, the skin, etc. In all these cases it was shown that for minimal resources of hardware and time, there was an increase in the effectiveness of decisions in the case of observing sick patients with the corresponding pathology. This increase is the reason we can recommend the results for medical practice in decision-making support systems.

After testing the specified models on testing sets it was established that the prediction quality increased by $5 \%$ and early diagnostics by $4 \%$ in comparison with the previously published author's works [1-5].

\section{Acknowledgements}

We would like to thank Al-Balqa' Applied University for their support, DFG Germany for the initial data, South West State University, Russia, for clinical tests and The Higher Council for Science and Technology, Jordan for their logistic aid.

\section{References}

1. Al-Kasasbeh R (2012) Biotechnical measurement and softwaresystem controlled features for determining the level of psycho-emotional tension on man-machine systems by fuzzy measures. Advances in Engineering Software 45: 137-143.

2. Korenevskiy N, Alshamasin M, Al-Kasasbeh R, Krupchatnikov R, lonescu F (2015) Prediction and prenosological diagnosis of stomach diseases based on energy characteristics of acupuncture points. Int. J Modelling Identification and controlrol, Vol. 23, No. 1.pp,55-67.

3. Al-Kasasbeh R, Korenevskiy N, lonescu F, Alshamasin M, Kuzmin A (2012) 
Citation: Al-Kasasbeh RT, Korenevskiy N, Alshamasin M, Klionskiy D (2015) Bioengineering System for Prediction and Early Prenosological Diagnostics of Stomach Diseases based on Energy Characteristics of Bioactive Points with Fuzzy Logic. J Biosens Bioelectron 6: 182. doi:10.4172/21556210.1000182

Prediction and prenosological diagnostics of heart diseases based on energy characteristics of acupuncture points and fuzzy logic. Computer Methods in Biomechanics and Biomedical Engineering 15: 681-689.

4. Al-Kasasbeh RT, Korenevskiy N, lonescu F, Alshamasin M, Smith AP, et al. (2013) Application of fuzzy analysis with the energy condition of bioactive points to the prediction and diagnosis of gastrointestinal tract diseases. International Journal of Biomedical Engineering and Technology (IJBET) 11: 136-154.

5. Al-Kasasbeh R, Korenevskiy N, Alshamasin M, Klionskiy D, lonescu F Numerical software algorithms for monitoring control processes and correcting health by synthesis of hybrid fuzzy rules of decision-making on the basis of changes in energetic characteristics of biologically active points. Int J Modelling Identification and Control accepted for publication.

6. Korenevskiy N, Kuzmin A, lonescu F, Al-Kasasabeh R (2008) Developing of a generalised mathematical model for the selection of the acupuncture points towards and optimised design of biomedical equipment. 1st Part: Acupuncture biophysics. Section 1: model of interaction between the projection zone and internal organs. Report of the DFG-research stage.

7. Lee MS, Jeong SY, Lee YH, Jeong DM, Eo YG, et al. (2005) Differences in electrical conduction properties between meridians and non-meridians. Am J Chin Med 33: 723-728.

8. Lu WA, Tsuei JJ, Chen KG (1999) Preferential direction and symmetry of electric conduction of human meridians. Bilaterally symmetrical acupoints provide better conductance for a better "connection". See comment in PubMed Commons below IEEE Eng Med Biol Mag 18: 76-78.

9. Portnov FP (1980) Electropuncture reflexotheraphy, Russia

10. Prokhorov EF, Gonzalez-Hernandez J, Vorobiev YV, Morales-Sanchez E, Prokhorova TE, et al. (2000) Electrophysical characterization of biologically active points and human skin by in vivo impedance measurement". Engineering in Medicine and Biology Society, 2000. Proceedings of the 22nd Annual International Conference of the IEEE, Chicago.

11. Voll R (1961) Gelöste und ungelöste Probleme den Electroakupun?tur. Schriftenreihe des Zentralrerbandes der Ärtzte für Naturheilverfahren 5: 148-152.

12. Wang ZM, Tan YH, Su MY (2009) Analysis of acupuncture point signal using Wavelet Transform and higher-order statistical method. Apperceiving Computing and Intelligence Analysis, ICACIA 2009. International Conference, Chengdu, China.

13. Korenevskiy N, Kuzmin Al, lonescu F, Al-Kasasabeh R (2010) Hardware and software complex for reflex therapy. 2nd Part: Examples of synthesis of combined fuzzy decision rules for medical and psychological diagnosis. Report of the DFGresearch stage.

14. Al-Kasasbeh RT, Korenevskiy N, Alshamasin M, Ionescu F, Smith A (2012) Prediction of gastric ulcers based on the change in electrical resistance of acupuncture points using fuzzy logic decision making. Computer Methods in Biomechanics and Biomedical Engineering 16: 302-313.

15. Al-Kasasbeh RT, lonescou F, Korenevsky N, Alshamasin M (2012) Prediction and Prenosological Diagnostics of Heart Diseases Based on Energy Characteristics of Acupuncture Points and Fuzzy Logic. Computer Methods in Biomechanics and Biomedical Engineering 15: 1476-8259.

16. Al-Kasasbeh R, Korenevskiy N, lonescou F, Alshamasin M, Kuzmin A (2011) Synthesis of fuzzy logic for prediction and medical diagnostics by energy characteristics of acupuncture points. See comment in PubMed Commons below J Acupunct Meridian Stud 4: 175-182.

17. Kai Z (1987) Acupoints Research Committee of China Society of Acupuncture and Moxibustion: Brief Explanation of Acupoints of the 14 Regular Meridians, Beijing, China.

18. Lee S, Choi C, Soh K, Yoon G (2001) Measurement and analysis of optical properties of acupuncture points and channels. Industrial Electronics Proceedings ISIE 2001. IEEE International Symposium on Volume: 1, Pusan.

19. Korenevskiy NA, Krupchatnikov R, Seregin AP (2010) Theoretical Fundamentals Biophysics Acupuncture with Applications in Biology and Medicine and Ecology using Fuzzy Logic, Monograph, in Russian, p.521, Kursk State Technical University Press, Kursk.

20. Korenevskiy NA, Al-Kasasbeh RT, lonescu F, (2011) Determining the Level of Psycho-Emotional Tension on a Heterogeneous Rules of Fuzzy Output. Proceedings of CSCS-18,18 International Conference on Control System and Computer Science, Bucharest, Romania.
21. Korenevskiy NA, Ionescu F, Kuzmin AA, Al-Kasasbeh $T$ (2009) Using Fuzzy Logic for Prediction of Occurrence, Aggravation and Pre-Nosological Diagnostics of Osteochondrosis of a Backbone's Lumbar Region. Proc of CI 2009, IASTED International Conference on Computational Intelligency, Honolulu, Hawaii, USA

22. Korenevskiy N, Al-Kasasbeh R, lonescu F, Alshamasin M, Smith A (2012) Mega-conference on Biomedical Engineering. Proceedings of the 4th International Conference of the Development of Biomedical Engineering, Ho Chi Minh City, Vietnam.

23. Lazoura H, Cohen M, Lazoura E (1998) Do acupuncture points have different absorption properties to laser light than surrounding skin. Bioelectromagnetism Proceedings of the 2nd International Conference on1998, Melbourne, Australia.

24. Korenevskiy N, Krupchatnikov RA, Gorbatenko SA (2008) Generation of fuzzy network models taught on the basis of data structure for medical expert systems. Med Tekh : 18-24.

25. Klir GJ, Ute H, Clair S, Yuan B (1997) Fuzzy Set Theory Foundations and Applications. Prentice-Hall, Inc.

26. Al-Kasasbeh RT, Korenevskiy NA, lonescu F, Kuzmin AA (2009) 'Synthesis of the combined fuzzy rules for medical applications with using tools of exploration analysis. Proceedings of the 4th International Conference on Interdisciplinary Approaches in Fractal Analysis (IAFA), Bucharest, Romania.

27. Korenevskiy NA, Bunyaev VV, Gadalov VN (2005) Synthesis of Models of Interaction between Internal Organs with Projective Zones and Use this Models in Reflexodiagnostics and Reflexotherapy, Kursk State Technical University, Kursk.

28. Kulback S (1959) Information Theory and Statistics. In: Wiley, New York London.

29. Fu LM, Shortliffe EH (2000) The application of certainty factors to neura computing for rule discovery. See comment in PubMed Commons below IEEE Trans Neural Netw 11: 647-657.

30. Zadeh L, (1996) Fuzzy sets, fuzzy logic, and fuzzy systems: selected papers. In Klir GJ, Yuan B (eds.). Advances in Fuzzy Systems-Applications and Theory.

31. Pal NR, Eluri VK, Mandal GK (2001) Fuzzy logic approaches to structure preserving dimensionality reduction. IEEE Transactions on Fuzzy Systems 10 277-286.

32. Verbruggen HB, Babuška R (1999) Fuzzy logic control advances in applications World Scientific Series in Robotics and Intelligent Systems 23, Technology and Engineering, Netherlands, Europe.

33. Zadeh LA (2005) Advances in Fuzzy Mathematics and Engineering:Fuzzy Sets and Fuzzy Information-Granulation Theory, Beijing Normal University Press, Beijing.

34. Al-Kasasbeh R, Korenevskiy N, lonescu F, Alshamasin M (2011) Prediction and prenosological diagnostics of gastrointestinal tract diseases based on energy characteristic of acupuncture points and fuzzy logic. In: Bioinformatics biomedical technology: Proceedings of the 3rd International Conference on Bioinformatics and Biomedical Technology (ICBBT 2011),Sanya, China.

35. Luvsan G (1991) Sketch of methods of east reflexotherapy, Russia 402. 